\title{
ECTOPIC GESTATION.
}

\author{
By W. J. SMYLY, M.D. ; \\ Master of the Rotunda Hospital.
}

[Read in the Section of Obstetrics, May 29th, 1891.]

Mr intention is to place on record and make a few observations upon three cases of extra-uterine pregnancy which $I$ have met with during the past year. Although so much attention has been devoted to this subject there are many points which still require elucidation, and the publication of individual cases is therefore still a matter of importance.

Extra-uterine foetation is generally classified as follows :-

I. Tubal-

a Tubal proper.

1 Intra-ligamentous.

2 Pedunculated.

$\beta$ Tubo-uterine, or interstitial.

$\gamma$ Tubo-abdominal.

$\delta$ Tubo-ovarian.

II. Ovarian.

III. Abdominal-

$\alpha$ Primary.

$\beta$ Secondary.

According to Mr. Tait, all cases are primarily tubal, but whether that be so or not I shall not now offer any opinion. My cases were all of that variety.

We cannot at present state with certainty what are the causes of this, which, far more than placenta prævia, deserves to be called "the greatest error of nature," and this cannot be greatly wondered at, since we know so little about the physiological process of im- 
pregnation. We do not even know how the ovum passes into or through the Fallopian tube, though I believe the majority of recent observers hold that it is due to the ciliary action of the epithelium lining the tubes. Neither do we know how the spermatozoa enter the uterus, nor whether they can under normal conditions pass up the tubes against the ciliary motion and actually reach the ovary. Mr. Lawson Tait believes that they cannot do so, and his theory as to the causation of ectopic gestation is a very simple one. $\mathrm{He}$ believes that the ovum is carried by the motion of the cilia through the tube into the cavity of the uterus, where it meets with the spermatozoa and becomes impregnated there. This view is also held by Wyder, who believes that normally impregnation occurs in the uterus alone. When, however, the cilia are destroyed by desquamative salpingitis the ovum is arrested in the tube, and the spermatozoa, being for the same reason permitted to enter, impregnate it in this position, where it becomes embedded in the mucous membrane thus prepared to form a nidus for it, and develops there.

This theory appears to me a plausible one, and explains the majority of cases, but there are some to which it does not seem to apply, as, for example, when extra-uterine pregnancy occurs in a woman who has previously enjoyed perfect health, and where the post-mortem examination reveals no inflammatory changes. I have not myself met with such a case, but they have been reported by Abel, Landau, and Klein. In Abel's case the patient, a healthy young woman, aged twenty-one, was suddenly seized with pain in the abdomen, symptoms of internal hæmorrhage, and abdominal collapse, of which she died fifteen minutes after medical aid was obtained, and therefore before abdominal section could be performed. The autopsy revealed a ruptured tube on the left side, but no signs whatever of inflammation. The tube on the opposite side was twisted three times upon its long axis, and on microscopic examination a diverticulum was also discovered. These conditions furnished two probable explanations of the occurrence of pregnancy in the opposite tube-namely, that the ovum had been arrested, either by the twisting of the tube, or in a diverticulum from it. This congenital twisting of the tube had been pointed out by Klob some 
years before as a possible cause of tubal pregnancy, but was originally described by Freund.

Hecker taught that the tube always burst during the early part of pregnancy, and Mr. Tait holds the same view, but this has been denied by Litzmann, Frænkal, Werth, and others. The rupture occurs either into the abdominal cavity or into the broad ligament; in the former case, according to Mr. Tait, the patient always bleeds to death, because the blood does not readily coagulate in the peritoneal cavity, but in this statement I cannot agree altogether, but would divide such cases into two varieties - those, namely, in which the rupture takes place into the normal peritoneal cavity, which I believe must almost inevitably prove fatal, unless the hæmorrhage be controlled by surgical interference, and those in which the intestines have become so agglutinated together by previous inflammation that a limited space only is left, which, becoming filled with blood, forms a hæmatocele, thus arresting the hæmorrhage.

When the tube bursts into the broad ligament the ovum may continue to develop outside the peritoneum-the "grossesse sousperitones pelvienne" of Deceimeris.

The diagnosis of extra-uterine gestation before rupture is generally difficult, but by no means impossible. When a patient presents herself with symptoms and signs of pregnancy, an enlarged and softened uterus, and a growing tumour beside it, the probabilities of the case being one of extra uterine pregnancy are very great. I have not, however, met with such a case, and can only confirm the statement of Mr. Lawson Tait, that in these countries, at least, medical aid is not sought until after rupture has occurred.

As to treatment. In the early months I have no doubt that, as Werth puts it, we should regard the enlarged tube as a malignant tumour requiring immediate extirpation, but after quickening I should follow Mr. Tait's practice, and wait until the child is viable; but I am not sure that the older practice of waiting longer still, until after the child's death, is not better, but this is a matter which future experience alone can decide. 
Case I.-Mrs. P. first came under my observation in November, 1887 , as an out-patient at the City of Dublin Hospital. She was then aged thirty; had been married ten years; had two children, the youngest of whom was seven years of age. After the birth of that child she had an inflammatory attack, and had been in ill-health ever since. She complained of menorrhagia, leucorrhœa, and pain.

The diagnosis was erosion of the cervix and endometritis. I curetted the uterus, and treated the erosion in the usual way.

In May, 1888, she became pregnant, and in August was attacked with violent pains and hæmorrhage; believing that she was threatening to abort, she remained in bed. Shortly after this she quickened, but experienced much greater discomfort during this than she had in former pregnancies. In November, 1888, she had a rigor, severe hæmorrhage, with expulsion of clots, great pain, and bearing-down sensation. She felt very ill, and was confined to bed for two or three weeks. She recovered, however, and went about again, but all foetal movements had ceased, the breasts became flaccid, menstruation returned, and continued ever since.

About Christmas she came to me again, telling me that the child was dead. She appeared to be in ill-health, and had a peculiar jaundiced complexion. Upon examination I found the uterus empty and but slightly enlarged, but there was a tumour on the right side but slightly movable, and in no way connected with the uterus.

In September last she came to me again at the Rotunda Hospital, and I examined her under chloroform, and could then distinctly feel the bones of the foetus through the walls of the tumour, which had also become decidedly smaller.

She was admitted into hospital, and an abdominal section performed on the 15th of October. The cyst was extra-peritoneal, and was enucleated from the cellular tissue without much difficulty. The cavity left was washed out, and a drainage tube inserted. The patient made a good recovery, and left the hospital a month after the operation.

The following points appear to be of interest in connection with this case. The patient, who for a time had been fruitful, acquired sterility in consequence of an inflammatory attack after childbirth, and having continued in this condition for seven years, became the subject of an extra-uterine gestation. This pregnancy evidently commenced in the right Fallopian tube, which burst about the third 
month, and after the ovum escaped into the sub-peritoneal tissue; it continued to develop between the layers of the broad ligament up to the sixth month, when it died and spurious labour occurred.

The yellow staining of the skin was probably due to urobilin resulting from the absorption of blood, and should have been an important guide to diagnosis.

The tumour is about the size of a cocoa-nut, and one hand of the foetus can be seen protruding upon the surface.

Case II.-G. P., aged twenty-four, unmarried, was admitted to the Rotunda Hospital, January 24, 1891. The patient had been treated previously in the Lock Hospital, and still showed signs of syphilis. She complained of pain in the hypogastrium, and a sanguineous discharge, which was accompanied by clots, and had continued for three weeks. On the evening of admission her temperature was $101 \cdot 8^{\circ} \mathrm{F}$.

Physical examination revealed a soft tumour (indistinguishable from the uterus), which I took to be that organ in the sixth month of pregnancy. The septic symptoms rapidly became worse, diarrhœa set in, and I determined to open the abdomen on the chance of discovering a cause. On opening the peritoneal cavity a large quantity of putrid blood flowed out. The intestines were so firmly matted together by former attacks of peritonitis, that it was a difficult and tedious task to reach the cornua of the uterus so as to secure the bleeding vessel. I say cornua because, unfortunately, having nothing to guide me I went to the wrong side first; but having finally secured both $I$ removed the appendages on the left, but found it impossible to do so on the right side, so I washed out the abdomen, and put a drainage tube into the sac, and another into Douglas' pouch. The woman, however, never rallied, and died the same evening.

CaSe III.-M. M., aged twenty-seven, married four and a half years, no children, no miscarriage, was sent to the Rotunda by Dr. Crosle, of Newry. Menstruation ceased in September, and all went well until the middle of January, when she was seized with sudden hæmorrhage and faintness. Hæmorrhage had continued on and off with distressing forcing down pain, and difficulty in micturition and defæcation. On examination a large hæmatocele was discovered displacing the uterus upwards and forwards above the pubes, and reaching upwards to midway between the pubes and 
the umbilicus. The diagnosis was retro-uterine hæmatocele, probably resulting from ruptured tubal pregnancy. All danger of death from hæmorrhage having passed, it was determined to await the absorption of the blood already effused. For three weeks all went well, but at the end of that time her temperature and pulse began to rise, and it was soon evident that the hæmatocele was becoming putrid. The tumour was, accordingly, laid open by a free incision through the vagina, and a quantity of putrid blood, clots, and a fotus about three inches long were evacuated. Following up the umbilical cord, the finger could reach the placenta, and, considering that the fotus had most probably been dead upwards of three weeks, I thought it would be a lesser risk to remove the placenta than to leave it behind, but the attempt was followed by such alarming hæmorrhage that $I$ was obliged to inject perchloride of iron to arrest it. A large drainage tube was inserted, and the cavity frequently washed out, but she died a few days afterwards from putrid absorption.

The unfortunate results in the two latter cases would tend to support the views of those who look upon an intra-peritoneal rupture of a tubal pregnancy as almost necessarily fatal, but I had met previously with three cases in which, though this accident had occurred, the blood being encysted, formed a retro-uterine hæmatocele, and all three made excellent recoveries.

My experience, therefore, amounts to this. I have seen, altogether, six cases. They all occurred in patients who had been sterile, or acquired sterility in consequence of pelvic inflammation. They were all tubal, and the tube burst within the first four months five times into the peritoneal eavity, and once into the broad ligament. In all the former cases the blood was encysted, forming a hæmatocele. None of them bled to death, but in two the blood decomposed, and septic absorption was the cause of death. The third case is most open to criticism. To have evacuated the cyst before the blood became putrid, and to have secured the bleeding vessel, would, very probably, have saved the patient's life, but that would involve operating on all intra-peritoneal hæmatoceles, and at present I am of opinion that they will do better if left alone. 